\title{
Design and Operation of Agriculture Based Pesticide Spraying Robot
}

\author{
Amruta Sulakhe ${ }^{1}$, M.N. Karanjkar ${ }^{2}$ \\ ${ }^{1}$ Department of Electronics and Telecommunication, Shree Tuljabhavani College of Engineering, Tuljapur, Osmanabad, India \\ ${ }^{2}$ Professor, Department of Electronics and Telecommunication, Shree Tuljabhavani College of Engineering, Tuljapur, Osmanabad , India
}

\begin{abstract}
Compared to spraying pesticides manually outdoors, the environment is more closed, and has a high temperature, humidity and so on for operating the spray work in the green-house. In order to protect laborer and reduce labor intensity, we develop a prototype of pesticide spraying robot specially used in the greenhouse. This paper emphatically describes the structural design, the robot control system design and the results of navigation experiment. The experiment shows that this robot can track a signal wire well which is set on the ground for navigation, and it obtains a nice steering ability even for short turns with the speed less than $0.5 \mathrm{~m} / \mathrm{s}$. Although the productivity of the prototype is not quite efficient, the robot still meets the requirements of pesticide spraying in the greenhouse without human operators.
\end{abstract}

Keywords: Android Smartphone, Bluetooth, Module, Robot, Single Microcontroller Chip

\section{Introduction}

Since the 70 s of last century, China has gradually popularized the plastic greenhouse and planting techniques, and has achieved significant economic and social benefits. Nowadays, China has become the largest country in greenhouse production. However, there are still some gaps in greenhouse production management and the level of automation compared with advanced countries. For example, in terms of production equipment in greenhouse, a large number of the small and medium greenhouses in china do the spraying work by using manual knapsack sprayer. Farmers have to work under a primitive protection, high temperature and humidity and poor ventilation condition for a long time. Compared to developed countries, particularly Japan, the average age of greenhouse manager is more than 65 years old.

Japanese greenhouse management has reached a high degree of automation and has been developing to a fully automated, unmanned direction. They have established the most advanced level of greenhouse production model in the world, called "plant factory ". The model adopts artificial supplementary light, and the whole production processes are controlled by computers. The cultivation is conducted by robots or robotic arms, so it is completely free from the binding of natural conditions [ $1-2$ ].Therefore, it is urgent to improve our green-house production equipment automation level and develop agricultural technique equipment to meet the requirements of the new era.

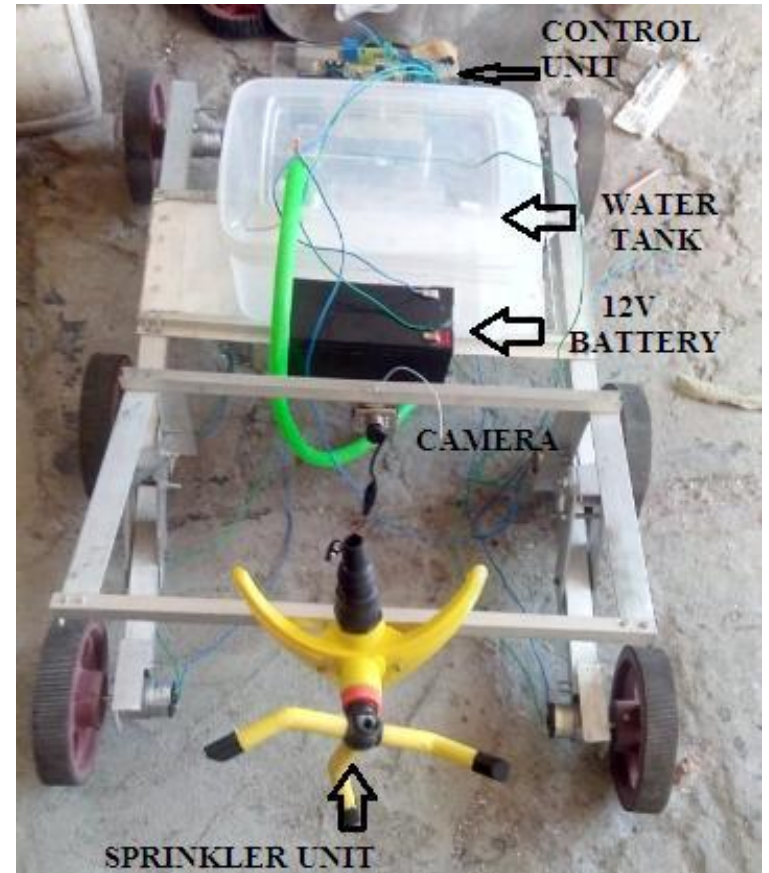

Figure 1: Pesticide spraying robot working in Agriculture

Nowadays smart phones are becoming more powerful with reinforced processors, larger storage capacities, richer entertainment function and more communication methods. Bluetooth is mainly used for data exchange; add new features to smart phones. Bluetooth technology, created by telecom vendor Ericsson in 1994[1], shows its advantage by integrating with smart phones. It has changed how people use digital device at home or office, and has transferred traditional wired digital devices into wireless devices. A host Bluetooth device is capable of communicating with up to seven Bluetooth mod-ules at same time through one link [2]. Considering its normal working area of within eight meters, it 


\section{International Journal of Science and Research (IJSR) \\ ISSN (Online): 2319-7064}

Index Copernicus Value (2013): 6.14 | Impact Factor (2014): 5.611

is especially useful in home environment. Thank for Bluetooth technology and other similar techniques, with dramatic increase in Smartphone users, smart phones have gradually turned into an all-purpose portable device and provided people for their daily use [3][4]. In recent years, an open-source platform Android has been widely used in smart phones [5].

Android has complete software package consisting of an operating system, middleware layer and core applications. Different from other existing platform like iOS (iPhone OS), it comes with software development kit (SDK), which provides essential tools and Application. Using a Smartphone as the brain of a robot is already an active research field with several open opportunities and promising possibilities. In this paper we present a review of current robots controlled by mobile phone and discuss a closed loop control systems using audio channels of mobile devices, such as phones and tablet computers. In our work, move the robot upward, backward, left and right side by the android application such as Arduino Bluetooth RC Car. This article is organized as follow: Section 2 describes the motivation of the work, Section 3 describes our experimental setup, Section 4 depicts a discussion about our experimental setup and Section 5 presents our conclusions.

\section{System Implementation}

\section{A. PIC16F877A Microcontroller}

The microcontroller to be used in the project is PIC 16F877 from Microchip family. There are numerous reasons behind selecting this microcontroller. It has 14 bit core, 40 pin DIP, works on up to $20 \mathrm{MHz}$ It also has flash memory for rewritable purpose. This microcontroller is very easy to be assembled, program and also the price is very cheap. The erasing time is almost unnoticeable because once new program are loaded into the PIC, the old program will automatically be erased immediately.

PIC16F877A already made with 368 bytes of Random Access Memory (RAM) inside it. Any temporary variables that are used inside the program are stored inside the RAM thus eliminating the need of external memory. The size of program code that can be stored is about 8k words inside PIC16F877A ROM. 1 word size is 14 bits which is more than enough for the system. The crystal oscillator speed that can be connected to the PIC microcontroller range from DC to $20 \mathrm{Mhz}$ [11].

\section{B. HC Serial Bluetooth}

$\mathrm{HC}$ Serial Bluetooth product consists of Bluetooth serial interface module and Bluetooth adapter. Bluetooth serial module is used for converting serial port to Bluetooth. This module has two modes: master and slaver device.

The device named after even number is defined to be master or slaver when out of factory and cant changed to the other mode. But for the device named after odd number, users can set the work mode (master or slaver) of the device by AT commands. HC-06 Specifically includes: Master device: HC06-M, M=Master Slaver device: HC-06-S, S=Slaver

The main function of Bluetooth serial module is replacing the serial port line, such as: One connects to Bluetooth master device while the other one connect to slaver device. Their connection can be built once the pair is made. This Bluetooth connection is equivalently liked to a serial port line connection including RXD, TXD signals. And they can communicate with each other.

1. When MCU has Bluetooth salve module, it can communicate with Bluetooth adapter of computer and smart phones. 2. The Bluetooth devices in the market mostly are salve devices, such as Bluetooth printer, Bluetooth GPS. So we can use master module to make pair and communicate with them. 3. Bluetooth serial modules operation doesnt need drive, and can communicate with the other Bluetooth device. But communication between two Bluetooth module require at two conditions: a) The communication must be between master and slave. b) The password must be correct.

\section{Wireless Camera}

Wireless cameras are basically described as a wireless transmitter carrying a camera signal. The Camera is wired to a wireless transmitter and the signal travels between the camera and the receiver. This works much like radio. The sound you hear on a radio is transmitted wirelessly and you tune to a certain frequency and hear the sound. Wireless cameras have a channel also. The receiver has channels to tune in and then you get the picture. The wireless camera picture is sent by the transmitter the receiver collects this signal and outputs it to your Computer OR TV Monitor depending on the receiver type.

Volume 4 Issue 12, December 2015 www.jijsr.net 


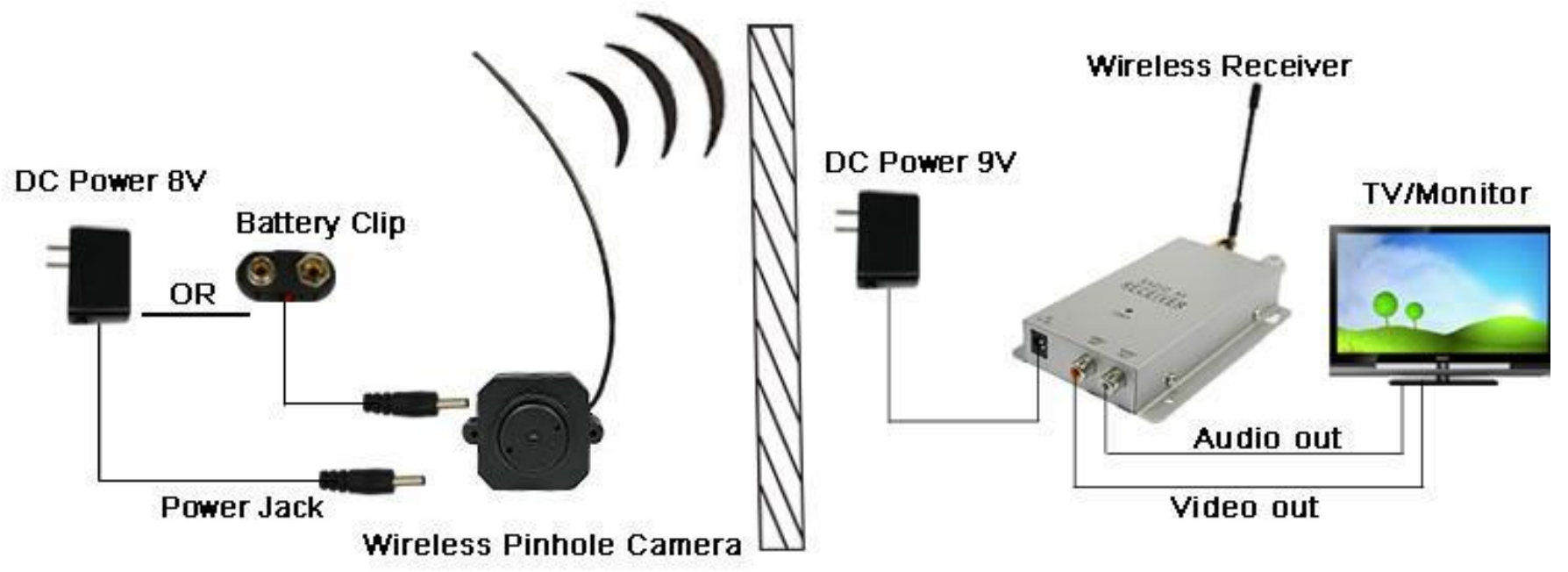

Figure 2: The wireless Camera / Transmitter

The Camera sees the image, the camera then provides the video to the transmitter, then the transmitter sends the wireless signal to the receiver. There are many types of wireless cameras. You can make most any camera wireless by adding a wireless transmitter and receiver. The camera and transmitter require power. The power is provided by battery and/ or transformer / adapter. The complete (Diagram 1) wiring for the wireless camera and transmitter end follows. The camera and transmitter both need power. The camera sees an image, and sends it to the transmitter, and the trans-mitter sends the signal out to the air. The receiver picks up the signal and outputs it to a TV / Computer / Digital Video recorder. This is a basic diagram. Many wireless cameras and transmitters are very small and the power is provided to both from one source. A good example of this is a hidden wireless camera. IE: A clock radio wireless camera is powered by plugging in the clock. The camera and wireless transmitter are provided power by the clock radio internally.

\section{Block Diagram}

A smart phone Android operated robot. Now here is a simple to control your robot/robo car using Bluetooth module HC-06 and PIC16F877A microcontroller with your android Smartphone device. The controlling devices of the whole system are a microcontroller. Bluetooth module, DC motors are interfaced to the microcontroller. The data receive by the Bluetooth module from android smart phone is fed as input to the controller. The controller acts accordingly on the DC motor of the robot. The robot in the project can be made to move in all the four directions using the android phone. The direction of the robot is indicators using LED indicators of the Robot system. In achieving the task the controller is loaded with program written using Embedded C Languages. Android smart phone controller Bluetooth robot using microcontroller is shown in figure 2 .

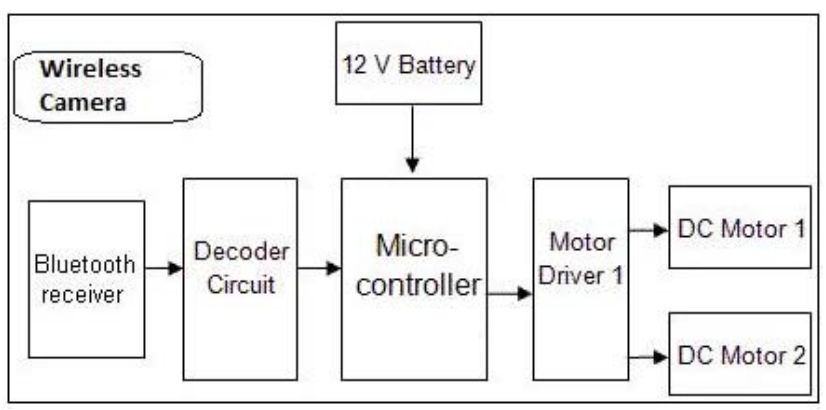

Figure 3: Block diagram of proposed system

\section{Conclusion}

The experiment showed that the robot can basically com-plete the work of automatic controlled and meet spraying requirements in the greenhouse. The control system has good stability and reliability. The wireless camera bases tracking performs well when the robot runs less than $0.5 \mathrm{~m} / \mathrm{s}$ and turning radius more than 0.5 meters. The spraying part can adjust po-sition within a certain range according to the height of target, and reduce leakage spray and heavy spray as much as possible. There are still some shortcomings in the robot system. For example, there is no location system for nozzle, some manual instructions are also required for remote control when the robot works. The system hasnt automatically adjusting devices of the spray quantity. In the current condition of rapid development of precision farming technology, we should make more efforts to realize variable automatic spray on the target.

\section{References}

[1] Sammons P J, Furukawa T , Bulgin A. Autonomous Pesticide Spraying Robot for Use in A Greenhouse[A]. Australian Conference on Robotics and Automation, Sydney Australian 2008.

[2] Zhang Ying, Mu Nan, Zhang Xueqin. The Development

Volume 4 Issue 12, December 2015 


\section{International Journal of Science and Research (IJSR) \\ ISSN (Online): 2319-7064}

Index Copernicus Value (2013): 6.14 | Impact Factor (2014): 5.611

Status and Trends of Facility Agriculture Overseas[J].

[3] Bluetooth based android phone/tablet controlled robot, http://www.robokits.co.in and http://www.robokitsworld.com

[4] R. Eaton, J. Katupitiya, K. W. Siew, and B. Howarth, Autonomous farming: Modeling and control of agricultural machinery in a unified framework, in Proc. 15th Int. Conf. Mechatronics and Machine Vision Practice, Auckland, New Zealand, Dec. 2008, vol. 1, pp. 499504.

[5] Simon Blackmore, Bill Stout, Maohua Wang, Boris Runov, Robotic Agriculture The future of agricultural mechanisation? AgroTechnology The Royal Veterinary and Agricultural University Agrovej 10 DK-2630 Taastrup, Denmark.

[6] B. Astrand and A. Baerdveldt, A vision based rowfollowing system for agricultural field machinery, Mechatronics, vol. 15, no. 2, pp. 251269, 2005.

[7] Potts, J. and Sukittanon, S. (2012) Exploting bluetooth on android mobile mobile devices for home security application, proceedings of southeastcan, 15-18 March 2012, orlando, florida,USA.

[8] Pavan.C, Dr. B. Sivakumar Wi-Fi Robot Video Surviellance Monitoring System International Journal of Scientific and Engineering Research Volume 3, Issue 8, August-2012 1 ISSN 2229-5518.

Volume 4 Issue 12, December 2015 www.ijsr.net 